\title{
Non-Uniform Constellations for Polarization Division Multiplexed CO-OFDM Systems
}

\author{
Dario PEREZ-CALDERON ${ }^{1,2}$, Vicente BAENA-LECUYER ${ }^{1}$, Ana Cinta ORIA ORIA ${ }^{1}$, \\ Jose GARCIA DOBLADO ${ }^{1}$
}

\author{
${ }^{1}$ Dept. of Electronic Engineering, University of Seville, Camino de los Descubrimientos S/N, 41092, Seville, Spain \\ ${ }^{2}$ Dept. of Television and Metrology, Bonch-Bruevich State University of Telecommunications, Bolshevikov Ave. 22, \\ Saint Petersburg, Russia
}

\{dperez, baena, cinta.oria, josegardo\}@gie.esi.us.es

Submitted October 26, 2020 / Accepted March 20, 2021

\begin{abstract}
In this paper we propose a transmission scheme for Coherent Optical Orthogonal Frequency Division Multiplexing (CO-OFDM) systems with Multiple Input - Multiple Output (MIMO) processing. Our proposal consists of the concatenation of two techniques, Non-Uniform Constellations (NUC) in the mapper, and Spatial Multiplexing (SM) in the implementation of Polarization-Division Multiplexed (PDM) systems. The main target of the proposed scheme is to reduce the overall performance loss introduced by Polarization Mode Dispersion (PMD) and Polarization Dependent Loss (PDL) in PDM-CO-OFDM systems. This approach will be compared to techniques traditionally used in CO-OFDM links as Golden codes and Silver codes as well as traditional SM. The full transmission chain has been modelled using Matlab. Simulations have been run to check the performance improvement achievable by our proposal showing a gain of up to $0.82 \mathrm{~dB}$ in the carrier to noise compared to traditional schemes, with no additional hardware complexity at the receiver side.
\end{abstract}

\section{Keywords}

MIMO, coherent optical OFDM systems, non-uniform constellations, polarization mode dispersion, polarization dependent loss

\section{Introduction}

Nowadays communication systems must face each time more demanding requirements to fulfil the user's necessities which are causing a growing demand of capacity in long haul optical communication networks. In order to prevent the capacity crunch in current backbone optical networks, it is necessary to optimize each block of the transmission chain and to use cutting edge technologies to achieve a capacity as close as possible to the theoretical limit set by Shannon [1], and the non-linear Shannon limit [2] in optical fibers.
Several schemes are being studied as possible candidates: the use of Multiple Input - Multiple Output (MIMO) techniques in optical channels [3], the implementation of new coded modulation schemes, like higher-order or multidimensional modulation formats [4], [5], or constellations shaping techniques [6], [7].

Two main MIMO schemes are currently being studied for optical communication systems [3]: PolarizationDivision Multiplexing (PDM) systems that can be implemented in current single-mode fiber (SMF) optical links, and Space-Division Multiplexing (SDM) systems that would require to install new fibers supporting multiple spatial modes as multi-mode fibers (MMFs) or multi-core fibers (MCFs). Although the initial studies of MIMO were focused on wireless propagation conditions, the optical channel also shows good conditions for these kinds of techniques to offer an overall system performance gain: multipath propagation due to the different propagation modes, orthogonal polarization modes that exchange power due to the propagation through the optical fiber, Polarization Dependent Loss (PDL) and Polarization Mode Dispersion (PMD).

Regarding constellations shaping schemes, recent advances have been also introduced in fiber-optic communications [7]: probabilistic shaping (PS) [6], geometric shaping (GS) [8], and hybrid probabilistic-geometric shaping (HPGS) [9] schemes.

In this paper we present a PDM-CO-OFDM (Polarization-Division Multiplexing Coherent Optical Orthogonal Frequency Division Multiplexing) system based in Spatial Multiplexing (SM) and Non-Uniform Constellations (NUC) instead of the conventional square M-ary Quadrature Amplitude Modulation (M-QAM) constellations for the Bit Interleaving Coding and Modulation (BICM) block. The NUC [10] is a particular scheme of constellation design techniques via geometric shaping, which have been adopted in recent terrestrial broadcasting standards. For example, the one-dimensional NUCs (1D-NUCs) family was included in the Digital Video Broadcasting Next Generation 
Handheld (DVB-NGH) standard [11], and two-Dimensional NUCs (2D-NUCs) were introduced in the newest digital terrestrial broadcasting system, ATSC 3.0 [12].

To the best of our knowledge, there are no publications in the literature addressing the implementation of both schemes (NUCs and SM) in optical communications. Our proposal will be compared to traditional MIMO diversity schemes used in fiber-optic communications, that is, Golden and Silver codes.

The rest of the paper is arranged as follows. Section 2 presents the scheme of the whole communication system, describing its functional blocks. Section 3 is dedicated to the fiber-optic channel model. Section 4 reviews the MIMO techniques to be compared to our proposal. Section 5 describes the basic principles of NUCs. In Sec. 6 we present our proposed modulation scheme. Section 7 shows the simulation results obtained, that is, the optimization of the constellations shape and the achievable gains for different system configurations. Finally, Section 8 summarizes the main conclusions that can be extracted from our work.

\section{System Overview}

In order to check the performance improvement achievable by our proposed technique, an end-to-end PDM-COOFDM baseband transmission chain model will be used (see Fig. 1).

The first block in the communication system is the Low Density Parity Check (LDPC) encoder [13]. LDPCs are Forward Error Correction (FEC) codes that reach a capacity close to the theoretical Shannon limit [1]. They are employed in communication systems like ATSC 3.0 [12] and DVB-NGH [11]. This last one uses code rates (CR) ranging from $3 / 15$ to $11 / 15$, with a codeword length of 16200 bits. These are the ones that we will use in our system.

After the LDPC encoder a bit interleaving process is carried out. It is known that the FECs work in optimal conditions when the errors do not affect consecutive bits and are uniformly spread along the codeword. This interleaving stage aims to get this purpose. Then, the interleaved bits are mapped into M-QAM constellations, in this case traditional square QAM constellations or NUC (this technique will be carefully explained in Sec. 5).

The mapped constellation points are processed by the MIMO block. Here the different techniques explained in Sec. 4 are applied. In this paper we use two orthogonal polarization modes $(2 \times 2$ MIMO scheme $)$ to exploit the capacity available in the optical fiber. After this block two parallel streams will be symmetrically processed. A frequency interleaving process is carried out for improved interleaving depth, to get an optimal performance of the FEC.

The OFDM block performs an Inverse Fast Fourier Transform (IFFT) of 256 points. The last block of the transmitter is the one performing the addition of the Cyclic Prefix.
The resulting signal in time is propagated through an optical fiber link, whose model is explained in Sec. 3. At the receiver side the reverse operations to the ones performed at the transmitter are carried out to recover the original information. The only additional step in the receiver is the channel estimation that can be accomplished by training sequences or using pilot patterns in the data frame. In this paper ideal channel estimation will be assumed.

\section{Optical Channel Model}

We consider a fiber link consisting of $N$ identical spans of length $L$, each supporting two polarization modes, and each terminated with an optical amplifier. Taking only into account PMD and PDL the channel can be modelled as a concatenation of random PMD and PDL elements [14] as seen in Fig. 2.

Following Jones' formalism [15], the Jones' matrix representing the $j$ th PMD element can be described as

$$
\mathbf{T}_{j}^{\mathrm{PMD}}(\omega)=\mathrm{e}^{-\mathrm{i} \overrightarrow{\boldsymbol{\beta}}_{j} \omega \frac{\vec{\sigma}}{2}}
$$

where $\overrightarrow{\boldsymbol{\sigma}}$ are the Pauli matrices:

$$
\boldsymbol{\sigma}_{x}=\left[\begin{array}{ll}
0 & 1 \\
1 & 0
\end{array}\right], \boldsymbol{\sigma}_{y}=\left[\begin{array}{cc}
0 & -\mathrm{i} \\
\mathrm{i} & 0
\end{array}\right], \boldsymbol{\sigma}_{z}=\left[\begin{array}{cc}
1 & 0 \\
0 & -1
\end{array}\right]
$$

and $\overrightarrow{\boldsymbol{\beta}}_{j}$ is a three dimensional (3D) vector whose direction represents the two polarization modes, and its modulus is the birefringence of the $j$ th span. We will consider the birefringence independent of the wavelength and therefore equal to the differential group delay (DGD) between the two propagation modes.

On the other hand, the $j$ th PDL element can be modelled as:

$$
\mathbf{T}_{j}^{\mathrm{PDL}}=\mathrm{e}^{-\mathrm{i} \overrightarrow{\boldsymbol{\alpha}}_{j} \frac{\vec{\sigma}}{2}}
$$

where $\vec{\alpha}_{j}$ is a $3 \mathrm{D}$ vector with the direction of the two polarization modes, and its modulus is the loss coefficient $\alpha_{j}$ defined by the differential loss between the two polarization modes:

$$
\mathrm{e}^{2 \alpha_{j}}=\frac{I_{\max }}{I_{\min }}
$$

where $I_{\max }$ and $I_{\min }$ are the intensities of the polarization modes with lowest and highest loss respectively.

The polarization states at the output $\left(\psi_{\text {out }}\right)$ and the input $\left(\psi_{\text {in }}\right)$ of the fiber link are related by the equation:

$$
\psi_{\mathrm{out}}=\mathbf{T}(\omega) \psi_{\mathrm{in}}
$$

where

$$
\mathbf{T}(\omega)=\mathbf{T}_{N}^{\mathrm{PDL}} \mathbf{T}_{N}^{\mathrm{PMD}} \cdots \mathbf{T}_{2}^{\mathrm{PDL}} \mathbf{T}_{2}^{\mathrm{PMD}} \mathbf{T}_{1}^{\mathrm{PDL}} \mathbf{T}_{1}^{\mathrm{PMD}} .
$$




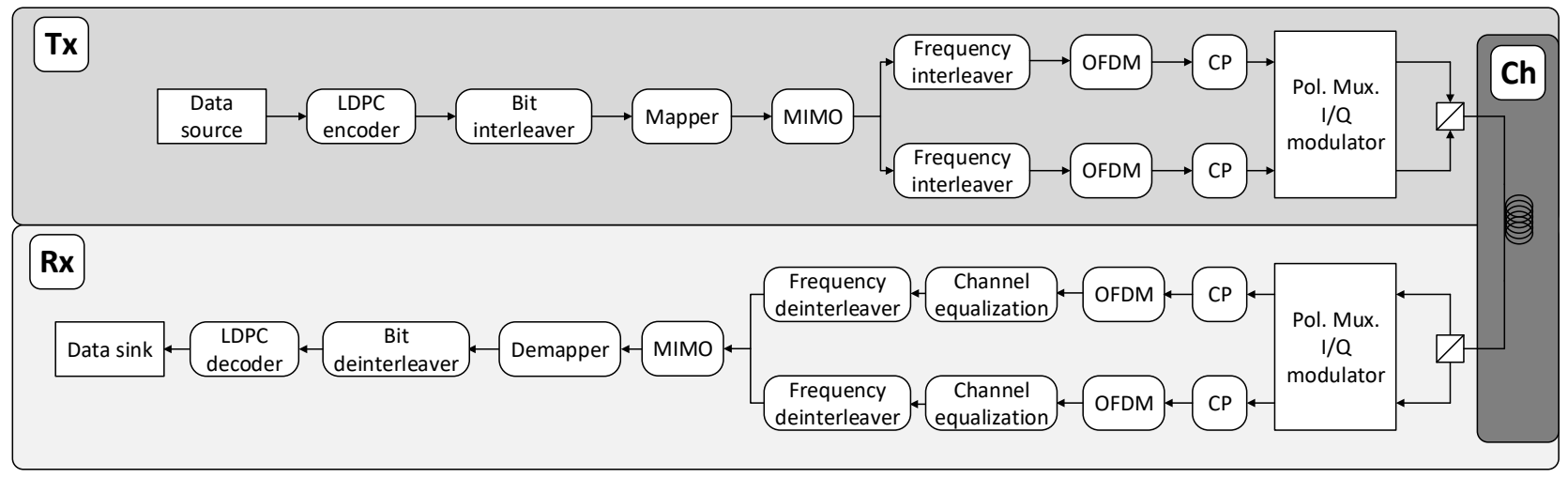

Fig. 1. PDM-CO-OFDM system diagram.

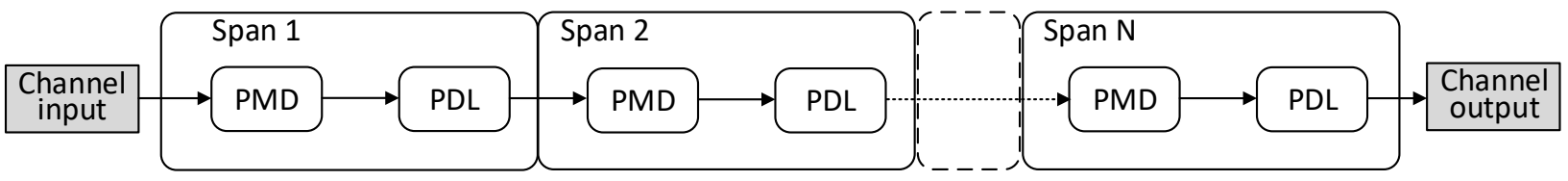

Fig. 2. Fiber link model.

It is known that the interaction of PMD and PDL elements introduces more sever effects than the one produced separately [15]. For example, the frequency dependence of PMD will turn global PDL of the optical link into a frequency dependent variable [16] and will even cause a loss of orthogonality of the polarization states [17] resulting in a significant impact on the overall system performance.

\section{MIMO Diversity Techniques}

The use of MIMO allows to greatly increase the capacity available for a given channel, surpassing the limit for traditional Single Input - Single Output (SISO) approaches. The first works in this promising field were carried out by Winters [18], Telatar [19], and Gans [20], and brought a high interest on what by the time was known as Smart Antenna Technologies. The main benefit of this scheme, and the reason why so much work has been carried out on this topic, is the high spectral efficiency offered in propagation scenarios with high dispersion, whose changes can be estimated accurately. The aforementioned high spectral efficiency is based on the fact that a channel with enough dispersion offers independent propagation paths for the information transmitted by every antenna in the system. In general, for an $M \times N$ MIMO system a capacity of $\min (M, N)$ channels can be achieved, where $M$ represents the number of transmitter antennas and $N$ the number of receiver ones. This makes the capacity to be scaled by $\min (M, N)$ when compared with the SISO equivalent system. This capacity gain requires a scattered propagation environment so that the channel matrix has full rank and can be estimated perfectly at the receiver side. The capacity of a MIMO channel strongly depends on the statistical and correlation properties of the propagation paths between antennas.

In order to reduce the negative effects of PMD and PDL, MIMO coding techniques can be applied to the signal. The codes used in this paper are widely studied in the literature so in this section we will have only a slight overview of their mathematical expressions and main characteristics.

\subsection{Golden Code}

Golden Code [21] is a space-time code for MIMO systems with two transmitter and receiver antennas. The generation of the code follows the expression

$$
\begin{gathered}
\mathbf{X}\left(s_{1}, s_{2}, s_{3}, s_{4}\right)= \\
\frac{1}{\sqrt{5}}\left[\begin{array}{cc}
(1+\sigma \mathrm{i}) s_{1}+(\theta-\mathrm{i}) s_{2} & (1+\sigma \mathrm{i}) s_{3}+(\theta-\mathrm{i}) s_{4} \\
(i-\theta) s_{3}+(1+\sigma \mathrm{i}) s_{4} & (1+\theta \mathrm{i}) s_{1}+(\sigma-\mathrm{i}) s_{2}
\end{array}\right]
\end{gathered}
$$

where $s_{1}, s_{2}, s_{3}$, and $s_{4}$ represent two consecutive $M$-QAM constellation symbols to be transmitted in two consecutive time instants, $\theta=(1+\sqrt{5}) / 2$ (the golden number that gives name to the codification), and $\sigma=1-\theta$.

The main properties of this code are: full rank, full rate, it reaches the frontier of diversity in multiplexing gain, its spectral efficiency is $2 \log _{2}(M) \mathrm{bits} / \mathrm{s} / \mathrm{Hz}$, and the number of distances to be computed for Maximum Likelihood (ML) detection is $M^{4}$, being $M$ the order of the base QAM modulation. 


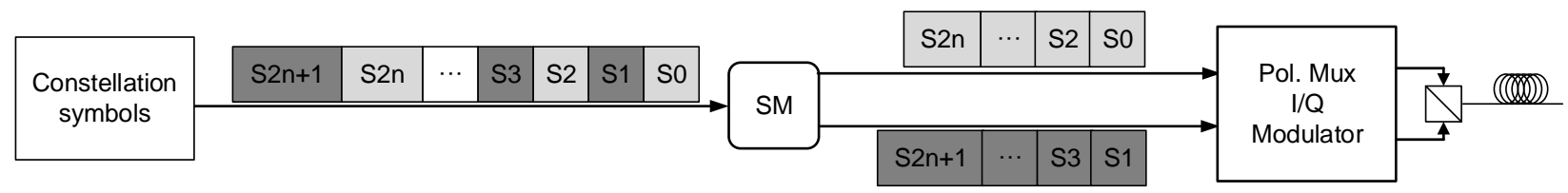

Fig. 3. SM modulation scheme.

\subsection{Silver Code}

Silver Code [22] is a space-time code for MIMO $2 \times 2$ systems that allows a simpler detection than Golden Code. The codification of the codewords follows the expression

$$
\mathbf{X}=\mathbf{X}_{a}\left(s_{1}, s_{2}\right)+\mathbf{T} \mathbf{X}_{b}\left(z_{1}, z_{2}\right)
$$

where

$$
\begin{gathered}
\mathbf{X}_{a}\left(s_{1}, s_{2}\right)=\left[\begin{array}{cc}
s_{1} & -s_{2}^{*} \\
s_{2} & s_{1}^{*}
\end{array}\right], \\
\mathbf{X}_{b}\left(z_{1}, z_{2}\right)=\left[\begin{array}{cc}
z_{1} & -z_{2}^{*} \\
z_{2} & z_{1}^{*}
\end{array}\right], \\
{\left[\begin{array}{l}
z_{1} \\
z_{2}
\end{array}\right]=\mathbf{U}\left[\begin{array}{l}
s_{3} \\
s_{4}
\end{array}\right]=\frac{1}{\sqrt{7}}\left[\begin{array}{cc}
1+\mathrm{i} & -1+2 \mathrm{i} \\
1+2 \mathrm{i} & 1-\mathrm{i}
\end{array}\right]\left[\begin{array}{l}
s_{3} \\
s_{4}
\end{array}\right],} \\
\mathbf{T}=\left[\begin{array}{cc}
1 & 0 \\
0 & -1
\end{array}\right]
\end{gathered}
$$

where $s_{i}$ represents a $M$-QAM symbol to be transmitted. This codification technique has very similar characteristics to the ones of the Golden Code, being its main ones: full rank, full rate, it reaches the frontier of diversity in multiplexing gain, the spectral efficiency is $2 \log _{2}(M)$ bits $/ \mathrm{s} / \mathrm{Hz}$, the number of distances to be computed for ML detection is $2 M^{3}$.

\subsection{Spatial Multiplexing (SM)}

The SM based codes only make use of the degree of freedom provided by space (multiple polarization modes in optical fibers). This eases the detection process, all of them have to compute $M^{2}$ distances for ML detection. The simplest one for a $2 \times 2 \mathrm{MIMO}$ configuration consists in sending the even symbols through a polarization mode and the odd ones through the other one as shown in Fig. 3.

\section{Non-Uniform Constellations (NUC)}

Traditionally, communication systems have used square $M$-QAM constellations as a modulation choice for the data bits in the BICM. However, Shannon stated in [1] that the maximum available capacity of a given Additive white Gaussian noise (AWGN) channel can be only obtained if the received signal has Gaussian distribution. Following this idea NUC tries to adopt a more Gaussian distribution shape in order to maximize the achievable capacity in the system. The
NUC approach used in this paper is the one known as One Dimensional Non-Uniform Constellations (1D-NUC), whose basic principles were settled in [10].

In NUC, symbols are optimized by using signal shaping techniques and this optimization depends on the carrier to noise ratio $(\mathrm{C} / \mathrm{N})$ and the propagation channel. This signal shaping leads to a more Gaussian shape of the constellation and, according to Shannon's theory, closer to the optimal usage of the channel capacity. However, studies as the one carried out in [23] show that the shaping gain obtained in discrete constellations in AWGN channels cannot exceed $1.53 \mathrm{~dB}$ (being this achievable only for high order constellations working at a high $\mathrm{C} / \mathrm{N}$ ) when compared to square constellations.

The gain obtained comes at a cost, an increased complexity in the demapping process. In general, for an ML demapping the complexity is proportional to the number of Euclidean distances to be computed from the received point to all the ideal constellation points. When using ML demapping on square Gray coded QAM constellations the $I$ and $Q$ components can be treated independently because there is no correlation between them, and thus the constellation can be considered as two orthogonal pulse amplitude modulations (PAM). For a Gray coded QAM constellation with $M=2^{b p s}$, where bps represents the number of bits per mapped symbol, the complexity of the demapping process is proportional to $2^{\frac{b p s}{2}+1}$. Moreover, the distances to be computed have only one dimension. If the constellation loses its square shape or its orthogonality to the real and imaginary axes, the assumption taken to simplify the demapping process is no longer valid. The distances to all the ideal points of the constellation need to be computed, and these distances will be two dimensional ones, leading to a complexity proportional to $2^{b p s}$. This is the case of 2D-NUC, however 1D-NUC maintains a square shape as can be seen in Fig. 4 and the two PAMs simplification can still be applied.

\subsection{D-NUC}

The difficulty of the optimization process for 1D-NUC depends on the available degrees of freedom, which depends on the constellation order, $M$, as follows [10]: $\sqrt{M} / 2-1$. This means that, for example, in a 16 symbols constellation (comparable with a 16-QAM) there is only one degree of freedom. Continuing with the example, for a 16QAM constellation, and before the energy normalization step, we have in each axis (real and imaginary) the following possible values: $-3,-1,1,3$. This leads to a constellation as the one shown in Fig. 4 (represented with circles). 


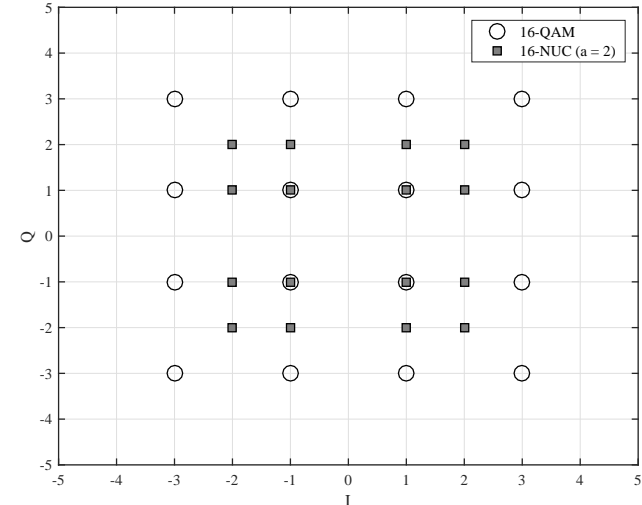

Fig. 4. 16-QAM vs 16-NUC $(a=2)$, without energy normalization.

To have a 1D-NUC we need to use the available degree of freedom and change the values that the constellation points can take in every axis by $-a,-1,1, a$. For $a=1$ the external points of the constellation overlap inner ones, and for $a=3$ the resulting constellation would be the previously defined 16-QAM. As it can be seen in Fig. 4 the inner points of the constellation remain unchanged, and the outer points come closer or farther to them depending on whether $a<3$ or not. As stated earlier, after choosing an appropriate value for $a$, the energy of the resulting constellation must be normalized. The values of $a<1$ are usually not considered because outer points would swap their position in the axis with inner ones and between them, and this would lead to a suboptimal behaviour due to the loss of the Gray mapping of the bits.

\section{Proposed Technique: NUC-SM}

In this paper we propose a combination of NUCs and SM (NUC-SM): first the data bits are mapped into a NUC and then, an SM process is carried out. In order to achieve optimal performance the optimization of the constellation shaping parameter, $a$, is needed. As stated in Sec. 5, the optimal value of $a$ depends on the working $\mathrm{C} / \mathrm{N}$, and so depends on the code rate used in the LDPC decoder (from $3 / 15$ to $5 / 15$ ). This will be achieved by means of computer simulations in the next section.

\section{Simulation Results}

The proposed modulation technique and the other ones exposed in this paper have been tested in a baseband OFDM system mathematically modelled using MATLAB together with mex C/CUDA functions to speed up the simulations. The main blocks are adapted from the Common Simulation Platform (CSP) [24], developed by the simulation group of the Technical Module of the Digital Video Broadcasting Project (TM-T2). The CSP was used by the DVB Project as validation tool for Digital Video Broadcasting Terrestrial Second Generation (DVB-T2) standard compliant systems [27]. The performed simulations can be divided in two groups: shaping parameter optimization and performance comparison. For these two groups the system configuration was set to a bandwidth of $12.0 \mathrm{GHz}$, fiber length of $2000 \mathrm{~km}$, no attenuation neither chromatic dispersion, PMD of $0.5 \mathrm{ps} / \sqrt{\mathrm{km}}$, and 20 PDL elements.

The total PDL of the system was set to three different values: 1, 2, and $3 \mathrm{~dB}$ (which can be found in installed links [25]) to represent optimistic, average and pessimistic scenarios. The values of the individual PDL elements are derived from the total PDL of the link following the procedure shown in [26]. All the results presented in this paper assume ideal channel estimation and ML detection. The interval of confidence set for the simulations was $99 \%$, leading to a minimum of 5000 different channel seeds per $\mathrm{C} / \mathrm{N}$ step at a bit error rate (BER) level close to $10^{-4}$.

\subsection{Shaping Parameter Optimization}

In order to find the optimum value of the shaping parameter, $a$, simulations were run for all the selected code rates $(3 / 15,4 / 15$ and 5/15). BER versus $\mathrm{C} / \mathrm{N}$ curves have been obtained for different values of $a$, ranging from 1 to 4 in steps of 0.2 , maintaining the same system configuration. The optimum value of $a$ has been selected as the one giving the lowest $\mathrm{C} / \mathrm{N}$ at a BER of $10^{-4}$. This process has been carried out for different values of total PDL (1, 2 and $3 \mathrm{~dB}$ ). As an illustrative example, and for the sake of brevity, Fig. 5 shows the difference in performance in $\mathrm{dB}$ of the different NUC constellations (different values of $a$ ) when compared to the standard 16-QAM $(a=3)$ for a total PDL of 1, 2 and $3 \mathrm{~dB}$, using a code rate of $4 / 15$.

The optimum values for the different configurations are summarized in Tab. 1. As it can be observed, there is a common tendency for every PDL simulated: lower values of $a$ perform better with lower CR fractions (better correction properties and so lower working $\mathrm{C} / \mathrm{N}$ ), whereas higher values of $a$ perform better with higher CR (lower correction properties and so higher working $\mathrm{C} / \mathrm{N}$ ).

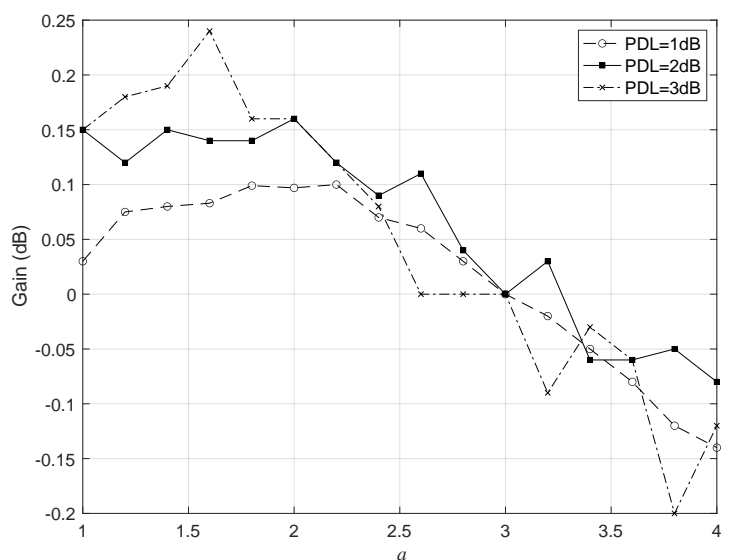

Fig. 5. Performance evolution of NUC depending on parameter $a$ compared to a standard 16-QAM constellation for a total PDL of $1 \mathrm{~dB}, 2 \mathrm{~dB}$ and $3 \mathrm{~dB}$, and code rate of $4 / 15$. 


\begin{tabular}{|c|c|c|c|}
\hline $\mathrm{CR}$ & $1 \mathrm{~dB}$ & $2 \mathrm{~dB}$ & $3 \mathrm{~dB}$ \\
\hline $3 / 15$ & 1.2 & 1 & 1.2 \\
\hline $4 / 15$ & 2.2 & 2 & 1.6 \\
\hline $5 / 15$ & 2.2 & 2.4 & 1.8 \\
\hline
\end{tabular}

Tab. 1. Optimum values of $a$.

\subsection{Performance Results}

Several simulations have been run in order to obtain the performance gain of our proposed modulation scheme. For the system configuration exposed in Sec. 7, the different techniques described in Sec. 4 and 6 were tested looking for the $\mathrm{C} / \mathrm{N}$ that leaded to a BER of $10^{-4}$ after the LDPC decoder. The simulations were run for a total PDL of 1,2 and $3 \mathrm{~dB}$, and for LDPC code rates of $3 / 15,4 / 15$ and $5 / 15$. Figure 6 shows the BER vs $\mathrm{C} / \mathrm{N}$ curves for all the studied MIMO codifications, a code rate of $3 / 15$ and a total PDL of $1 \mathrm{~dB}$. As it can be seen our proposed technique offers a gain of $0.46 \mathrm{~dB}$ over SM and Silver code, and about $0.49 \mathrm{~dB}$ over Golden code, measured at a BER $=10^{-4}$.

Table 2 summarizes all the obtained results and shows the performance gain achievable by using our proposed NUCSM scheme. As it can be denoted, the performance gain obtained ranges from $0.01 \mathrm{~dB}$, in the worst case observed, to $0.82 \mathrm{~dB}$. From these results a clear tendency can be observed: the performance gain is higher for lower code rate fractions and for higher PDL. Lower code rate fractions operate at a lower $\mathrm{C} / \mathrm{N}$ leading to a noisier propagation scenario. From Tab. 1, it can be observed that the shaping parameter is lower for this kind of propagation conditions, making the constellation points to be closer. For higher code rates, and so higher working $\mathrm{C} / \mathrm{N}$, the optimum shape is closer to the original QAM.

It is worth noting that the capacity gain obtained comes to no additional hardware complexity when compared to SM schemes with square $M$-QAM constellations. The only additional step is changing the values of the constellation points in the complex plane but the complexity at the receiver when using ML detection remains the same, $M^{2}$.

\section{Conclusion}

In this paper we presented a MIMO modulation scheme for an optical fiber link. This scheme uses NUC, one of the most promising modulation techniques applied in cutting edge communications systems as DVB-NGH or ATSC3.0, in conjunction with SM, a well-known MIMO diversity technique. The proposed scheme has been compared to classical MIMO coding schemes as Golden and Silver codes, and SM, in a frame of a $2 \times 2$ PDM-CO-OFDM transmission system using traditional square $M$-QAM constellations. The important capacity gain obtained, up to $0.82 \mathrm{~dB}$ when compared to Golden code, comes to no additional hardware complexity at the receiver side when compared to SM, only requiring the definition of the reshaped constellations.

\begin{tabular}{|c|c|c|c|c|}
\hline \multirow{2}{*}{ PDL } & Code & $3 / 15$ & $4 / 15$ & $5 / 15$ \\
\hline \multirow{3}{*}{$1 \mathrm{~dB}$} & Silver & 0.46 & 0.08 & 0.02 \\
\cline { 2 - 5 } & Golden & 0.49 & 0.14 & 0.02 \\
\cline { 2 - 5 } & SM & 0.46 & 0.1 & 0.01 \\
\hline \multirow{3}{*}{$2 \mathrm{~dB}$} & Silver & 0.47 & 0.18 & 0.16 \\
\cline { 2 - 5 } & Golden & 0.57 & 0.13 & 0.17 \\
\cline { 2 - 5 } & SM & 0.45 & 0.16 & 0.08 \\
\hline \multirow{3}{*}{$3 \mathrm{~dB}$} & Silver & 0.68 & 0.45 & 0.28 \\
\cline { 2 - 5 } & Golden & 0.82 & 0.37 & 0.32 \\
\cline { 2 - 5 } & SM & 0.66 & 0.24 & 0.19 \\
\hline
\end{tabular}

Tab. 2. Performance gain (dB) of NUC-SM.

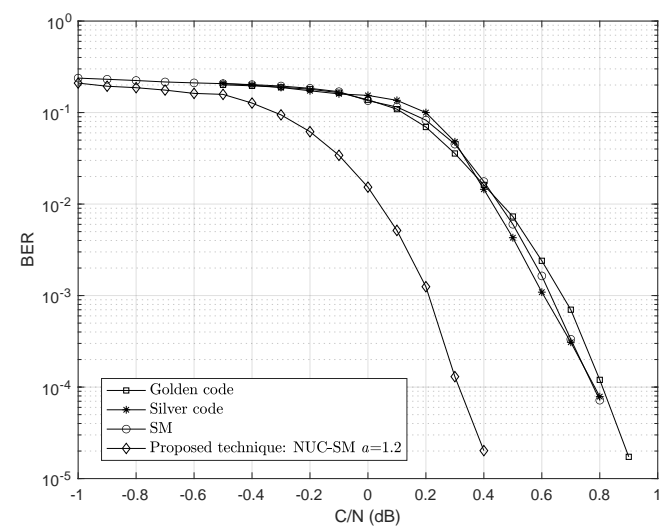

Fig. 6. Simulation results for $16-\mathrm{QAM}$, PDL $1 \mathrm{~dB}$ and code rate $3 / 15$.

\section{References}

[1] SHANNON, C. E. A mathematical theory of communication. Bell System Technical Journal, 1948, vol. 27, no. 3, p. 379-423 and p. 623-656. DOI: $10.1002 / \mathrm{j} .1538-7305.1948 . t b 01338 . x$

[2] ESSIAMBRE, R., KRAMER, G., WINZER, P.J., et al. Capacity limits of optical fiber networks. Journal of Lightwave Technology, 2010, vol. 28, no. 4, p. 662-701. DOI: 10.1109/JLT.2009.2039464

[3] WILlNER, A. E. Optical Fiber Telecommunications VII. Academy Press Elsevier, 2019. ISBN: 9780128165027

[4] WANG, Y., OKAMOTO, S., KASAI, K., et al. Single-channel $200 \mathrm{Gbit} / \mathrm{s}, 10 \mathrm{Gsymbol} / \mathrm{s}-1024$ QAM injection-locked coherent transmission over $160 \mathrm{~km}$ with a pilot-assisted adaptive equalizer. Optics Express, 2018, vol. 26, no. 13, p. 17015-17024. DOI: $10.1364 /$ OE.26.017015

[5] KARLSSON, M., AGRELL, E. Four-dimensional optimized constellations for coherent optical transmission systems. In Proceedings of the European Conference on Optical Communications (ECOC). Torino (Italy), 2010, p. 1-6. DOI: 10.1109/ECOC.2010.5621574

[6] CHO, J., CHEN, X., CHANDRASEKHAR, S., et al. Trans-atlantic field trial using high spectral efficiency probabilistically shaped 64-QAM and single-carrier real-time 250-Gb/s 16-QAM. Journal of Lightwave Technology, 2018, vol. 36, no. 1, p. 103-113. DOI: $10.1109 /$ JLT.2017.2776840

[7] QU, Z., DJORDJEVIC, I. B., ANDERSON, J. Two-dimensional constellation shaping in fiber-optic communications. Applied Sciences, 2019, vol. 9, no. 9, p. 1889-2001. DOI: 10.3390/APP900188 
[8] ZHANG, S., YAMAN, F. Design and comparison of advanced modulation formats based on generalized mutualinformation. Journal of Lightwave Technology, 2018, vol. 36, no. 2, p. 416-423. DOI: 10.1109/JLT.2017.2779753

[9] ZHANG, S., QU, Z., YAMAN, F., et al. Flex-rate transmission using hybrid probabilistic and geometric shaped 32QAM. In Proceedings of the Optical Fiber Communication Conference (OFC). San Diego (USA), 2018, p. 1-3. DOI: 10.1364/ofc.2018.m1g.3

[10] STOTT, J. CM and BICM Limits for Rectangular Constellations. BBC Research and Development, White paper 257, 2013.

[11] EUROPEAN TELECOMMUNICATIONS STANDARD INSTITUTE ETSI YORK. Digital Video Broadcasting (DVB), Next Generation broadcasting system to Handheld, physical layer specification (DVB-NGH). Draft ETSI EN 303105 V1.1.1, 2012.

[12] ADVANCED TELEVISION SYSTEMS COMMITTEE (ATSC). ATSC Standard: Physical Layer Protocol, document A/322:2020.

[13] GALLAGER, R. G. Low-density parity-check codes. IRE Transactions on Information Theory, 1962, vol. 8, no. 1, p. 21-28. DOI: 10.1109/TIT.1962.1057683

[14] GISIN, N., HUTTNER, B. Combined effects of polarization mode dispersion and polarization dependent losses in optical fibers. Optics Communications, 1997, vol. 142, no. 1-3, p. 119-125. DOI: $10.1016 / \mathrm{S} 0030-4018(97) 00236-8$

[15] HUARD, S. Polarization of Light (in French). Paris (France): Masson, 1993. ISBN: 978-2-225-84300-6

[16] LIMA, A. O., LIMA, I. T., MENYUK, C. R., et al. Comparison of penalties resulting from first-order and all-order polarization mode dispersion distortions in optical fiber transmission systems. Optics Letters, 2003, vol. 28, no. 5, p. 310-312. DOI: 10.1364/OL.28.000310

[17] MORI, K., KATAOKA, T., KOBAYASHI, T., et al. Statistics and performance under combined impairments induced by polarizationdependent-loss in polarization-division-multiplexing digital coherent transmission. Optics Express, 2011, vol. 19, no. 26, p. 673-680. DOI: $10.1364 / \mathrm{OE} .19 .00 \mathrm{~B} 673$

[18] WINTERS, J. On the capacity of radio communication systems with diversity in a Rayleigh fading environment. IEEE Journal on Selected Areas on Communications, 1987, vol. 5, no. 5, p. 871-878. DOI: $10.1109 /$ JSAC.1987.1146600

[19] TELATAR, I. E. Capacity of multi-antenna Gaussian channels. AT \& T Bell Laboratories, 1999, vol. 10, no. 6, p. 585-595. DOI: $10.1002 /$ ett.4460100604

[20] FOSCHINI, G. J., GANS, M. J. On limits of wireless communications in a fading environment when using multiple antennas. Wireless Personal Communications, 1998, vol. 6, p. 311-335. DOI: 10.1023/A:1008889222784

[21] BELFIORE, J. C., REKAYA, G.,VITERBO, E. The Golden Code: A $2 \times 2$ full-rate space-time code with non-vanishing determinants. IEEE Transactions on Information Theory, 2005, vol. 51, p. 1432-1436. DOI: 10.1109/TIT.2005.844069

[22] BIGLIERI, B., HONG, Y., VITERBO, E. On fast-decodable spacetime block codes. IEEE Transactions on Information Theory, 2009, vol. 55, no. 2, p. 524-530. DOI: 10.1109/TIT.2008.2009817

[23] FORNEY, G. D., WEI, L. F. Multidimensional constellations part: I: Introduction, figures of merit and generalized cross constellations. IEEE Journal on Selected Areas in Communications, 1989, vol. 1, no. 6, p. 877-892. DOI: $10.1109 / 49.29611$
[24] HAFFENDEN, O. The Common Simulation Platform. BBC Research, White Paper 196, 2011.

[25] NELSON, L. E., ANTONELli, C., MECOZZI, A., et al. Statistics of polarization dependent loss in an installed long-haul WDM system. Optics Express, 2011, vol. 19, no. 7, p. 6790-6796. DOI: 10.1364/OE.19.006790

[26] MECOZZI, A., SHTAIF, M. The statistics of polarization-dependent loss in optical communication systems. IEEE Photonics Technology Letters, 2002, vol. 14, no. 3, p. 313-315. DOI: 10.1109/68.986797

[27] EUROPEAN TELECOMMUNICATIONS STANDARD INSTITUTE ETSI YORK. Digital Video Broadcasting (DVB), Frame Structure Channel Coding and Modulation for a Second Generation Digital Terrestrial Television Broadcasting System (DVB-T2), ETSI EN 302 755 V1.2.1., 2011.

\section{About the Authors...}

Darío PÉREZ-CALDERÓN was born in Jerez de la Frontera, Spain. He received his Telecommunication Engineering degree in 2009, his Master degree in Electronics, Signal Processing and Communications in 2011, and his Ph.D. in 2017 from the University of Seville, Spain. Since 2008, he has been with the Department of Electronic Engineering, Higher Technical School of Engineering, University of Seville. From 2019 he is also with the Department of Television and Metrology of the Bonch-Bruevich State University of Telecommunications, Saint Petersburg, Russia. His research interest is in OFDM systems and diversity techniques for communication systems, especially the ones applied in DVB standards.

Vicente BAENA-LECUYER was born in Athis-Mons, France. He received his Telecommunication Engineering and Ph.D. degrees from the University of Seville (Spain) in 1997 and 2001, respectively. Since 1997, he has been with the Department of Electronic Engineering, Higher Technical School of Engineering, University of Seville. His current research interest is mainly in OFDM systems, especially in digital video broadcasting systems.

Ana Cinta ORIA ORIA was born in Huelva, Spain. She received her Master and Ph.D. degrees in Telecommunication Engineering in 2005 and 2010, both from the University of Seville. Since 2005, she has been with the Department of Electronic Engineering, Higher Technical School of Engineering, University of Seville. Her current research interest is in OFDM systems.

José GARCÍA DOBLADO was born in Huelva, Spain. He received his Telecommunication Engineering degree and $\mathrm{Ph} . D$. from the University of Seville in 2008 and 2013. Since 2006, he has been with the Department of Electronic Engineering, Higher Technical School of Engineering, University of Seville. His current research interest is mainly in digital signal processing, especially digital video broadcasting. 